\title{
ВОЗМОЖНОСТИ ПРИМЕНЕНИЯ НОВОЙ МОЛЕКУЛЯРНО-ГЕНЕТИЧЕСКОЙ ПАНЕЛИ В ДООПЕРАЦИОННОЙ ДИАГНОСТИКЕ ЗЛОКАЧЕСТВЕННЫХ ОБРАЗОВАНИЙ ЩИТОВИДНОЙ ЖЕЛЕЗЫ
}

\author{
Ромащенко П.Н., Майстренко Н.А., Криволапов Д.С., Симонова М.С. \\ ФГБВОУ ВО «Военно-медицинская академия имени С.М. Кирова» МО, Санкт-Петербург
}

Увеличение числа больных фолликулярными неоплазиями и раком щитовидной железы (ЩЖ) требует поиска новых методов диагностики, направленных на повышение точности предоперационной верификации злокачественных образований с целью определения наиболее рациональной тактики их лечения. Такими методами являются иммуноцитохимический и молекулярно-генетический, позволяющие определить патоморфологический тип опухоли, ее биологические свойства, степень агрессивности, риск рецидивирования и эффективность проводимого лечения.

ЦЕЛЬ: обосновать целесообразность применения новой молекулярно-генетической панели в дооперационной диагностике злокачественных образований щитовидной железы.

МАТЕРИАЛЫ И МЕТОДЫ: проведен ретроспективный анализ результатов обследования и хирургического лечения 60 больных новообразованиями ЩЖ. С целью уточнения характера образований и лечебно-диагностической тактики общепринятый алгоритм обследования был дополнен определением экспрессии онкомаркера Galectin-3 и натрий-йодидного симпортера (NIS) методом проточной флуороцитометрии и мутации V600E гена BRAF методом полимеразной цепной реакции в клеточном материале, полученном при пункционной тонкоигольной аспирационной биопсии. На первом этапе исследования осуществлена оптимизация дооперационной диагностики высокодифференцированного рака ЩЖ (ВДРЩЖ) посредством оценки уровня экспрессии Galectin-3 и выявления BRAF-мутации в пункционном материале у всех 60 испытуемых, на втором - изучено прогностическое значение экспрессии NIS в выявлении резистентности рака ЩЖ к радиойодтерапии (РЙТ) у 26 больных с гистологически подтвержденным ВДРЩЖ.

РЕЗУЛЬТАТЫ: анализ результатов применения новой молекулярно-генетической панели показал, что уровень экспрессии Galectin-3 выше 47,6\% позволяет с точностью до 91,7\% прогнозировать наличие у больного ВДРЩЖ, что может потребовать выполнения тиреоидэктомии даже при фолликулярной опухоли больших размеров, локализующейся в одной доле. Выявление соматической мутации V600E гена BRAF в клеточном материале опухоли с вероятностью до 97,1\% указывает на наличие папиллярного рака щЖ, который характеризуется более агрессивным течением и требует выбора радикального объема оперативного вмешательства - тиреоидэктомии с профилактической центральной лимфаденэктомией, даже при микрокарциномах. При этом у пациентов с фолликулярным раком и доброкачественными образованиями ЩЖ BRAF-мутация выявлена не была. На основании результатов определения уровня экспрессии NIS доказана возможность использования данного маркера в качестве прогностического в оценке эффективности РЙТ. Получено критичное значение экспрессии NIS для развития радиойодрезистентности ВДРЩЖ —<1,8\%, что требует выполнения у больных, нуждающихся в тиреоидэктомии, профилактической лимфаденэктомии ввиду повышенного риска развития рецидива даже после комбинированного лечения.

ВЫВоды: использование новой молекулярно-генетической панели в лечебно-диагностическом алгоритме у пациентов с новообразованиями щитовидной железы способствует повышению точности предоперационной морфологической верификации высокодифференцированного рака, выбору наиболее рационального варианта хирургического лечения в каждом конкретном случае в целях улучшения его результатов и снижения риска рецидивирования онкологического процесса. 